\title{
DKK1 inhibits proliferation and migration in human retinal pigment epithelial cells via the Wnt/ $\beta$-catenin signaling pathway
}

\author{
JINZI ZHOU ${ }^{1}$, JIAN JIANG $^{2}$, SHUHONG WANG $^{1}$ and XIAOBO XIA ${ }^{2}$ \\ ${ }^{1}$ Department of Ophthalmology, Huai'an First People's Hospital, Nanjing Medical University, Huai'an, Jiangsu 223300; \\ ${ }^{2}$ Department of Ophthalmology, Xiangya Hospital Central South University, Changsha, Hunan 410008, P.R. China
}

Received March 29, 2015; Accepted April 29, 2016

DOI: $10.3892 /$ etm.2016.3422

\begin{abstract}
Retinal pigment epithelial (RPE) cells play important roles in diabetic retinopathy (DR). Dickkopf 1 (DKK1) has been reported to be important in the regulation of cell proliferation and migration. However, there are few previous studies regarding DKK1 in RPE cells. Therefore, in the present study, we investigated the effect of DKK1 on the proliferation and migration of human RPE cells, and the signaling mechanisms underlying these effects. The results showed that the overexpression of DKK1 significantly inhibited the proliferation and migration of ARPE-19 cells. In addition, overexpression of DKK1 markedly inhibited the expression of $\beta$-catenin and cyclin D1 in ARPE-19 cells. Collectively, the present findings suggest that the overexpression of DKK1 inhibited the proliferation and migration of RPE cells by suppressing the Wnt/ $\beta$-catenin signaling pathway. Therefore, DKK1 are able to augment the growth of human RPE, and further studies are warranted to investigate the effects of DKK1 effects on DR.
\end{abstract}

\section{Introduction}

Diabetic retinopathy (DR) is a leading cause of blindness and vision loss in numerous countries (1). DR is a chronic, progressive, sight-threatening disease associated with prolonged hyperglycemia. Retinal pigment epithelial (RPE) cells play an important role in the development of DR. Uncontrolled proliferation and migration of RPE cells may form pathological membranes on both surfaces of the neural retina, which can result in visual impairment (2).

The Dickkopf (DKK) family is one of the Wnt antagonist families, and inhibits Wnt signaling by binding to the lipoprotein receptor-related protein (LRP) 5/6 component of

Correspondence to: Dr Jinzi Zhou, Department of Ophthalmology, Huai'an First People's Hospital, Nanjing Medical University, 6 Beijing Road West, Huai'an, Jiangsu 223300, P.R. China E-mail: jinzi_zhoujs@163.com

Key words: dickkopf 1, retinal pigment epithelial, proliferation, migration the Wnt receptor complex (3). It consists of four members, each of which possesses an N-terminal signal peptide and contains two conserved cysteine-rich domains separated by a linker region (4). Previous studies found that DKK1 markedly suppresses tumor growth by activating apoptosis of melanoma cells (5). In addition, DKK1 plays a critical role in the development of inflammatory arthritis. It has been reported that the protein expression level of DKK1 is significantly increased in the serum of patients with rheumatoid arthritis (6), and DKK1 regulates bone development and accrual and maintenance of bone mass (7,8). Recently, a study showed that plasma DKK-1 levels were significantly lower in DR patients compared with non-diabetic controls and non-DR (NDR) patients (9). Furthermore, DKK-1 levels were lower in proliferative DR (PDR) patients compared with non-proliferative PDR (NPDR) patients (9). However, there is no information regarding the effects of DKK1 in RPE cells. Therefore, in the present study, we investigated the effect of DKK1 on the proliferation and migration of human RPE cells, and the signaling mechanisms underlying these processes.

\section{Materials and methods}

Cell culture. Human ARPE-19 cell line was purchased from the American Type Culture Collection (Manassas, VA, USA). Cells were cultured in Dulbecco's modified Eagle's medium and Ham's F-12 nutrient mixture (DMEM/F12; Gibco, Rockville, MD, USA) supplemented with $10 \%$ fetal bovine serum (FBS; Santa Cruz Biotechnology, Inc., Santa Cruz, CA, USA), $100 \mathrm{U} / \mathrm{ml}$ penicillin and $100 \mathrm{U} / \mathrm{ml}$ streptomycin (Sigma-Aldrich, St. Louis, MO, USA) at $37^{\circ} \mathrm{C}$ in $5 \% \mathrm{CO}_{2}$ and 95\% humidity.

Construction of plasmids and transfection. All recombinant adenovirus were constructed as previously described (10). In brief, full-length DKK1 cDNA (Sangon, Shanghai, China) was amplified and subcloned into pAdTrack-cytomegalovirus (CMV; Invitrogen, Carlsbad, CA, USA), and green fluorescent protein (GFP; Sigma-Aldrich, St. Louis, MO, USA) was used as a control. Then, the shuttle plasmids pAdTrack-CMV (Invitrogen) and pAdEasy-1 were recombined in the Escherichia coli strain BJ5183 (Institute of Biochemistry and Cell Biology of the Chinese Academy of 
Sciences, Shanghai, China). The recombinant plasmids were transfected into 293 cells to generate recombinant adenovirus. The recombinant adenoviruses were harvested and the titers were determined using a p24 ELISA kit (Cell Biolabs, Inc., San Diego, CA, USA).

For in vitro transfection, ARPE-19 cells (American Type Culture Collection, Manassas, VA, USA) were seeded in each well of 24-well microplates, grown for $24 \mathrm{~h}$ to reach $50 \%$ confluence, and transfected with Ad-DKK1 or Ad-GFP using Lipofectamine 2000 (Invitrogen; Thermo Fisher Scientific, Inc., Carlsbad, CA, USA), according to the manufacturer's instructions.

Reverse transcription-quantitative polymerase chain reaction (RT-qPCR) analysis. Total RNA was extracted from ARPE-19 cells using TRIzol reagent (Invitrogen; Thermo Fisher Scientific, Inc.) following the manufacturer's protocol. Subsequently, $\sim 5 \mu \mathrm{g}$ total RNA was reverse transcribed into cDNA using M-MLV reverse transcriptase (Clontech Laboratories, Inc., Palo Alto, CA, USA). The following primers were used: DKK1 forward, 5'-GATCATAGCCCT TGGATGGG-3' and reverse, 5'-GGCACAGTCTGATGA CCGG-3'; $\beta$-actin forward, 5'-CCACCCATGGCAAAT TCCATGGCA-3' and reverse, 5'-TCTAGACGGCAGGTC AGGTCCACC-3'. A total of 1.25 units GoTaq Flexi DNA polymerase (Promega, Madison, WI, USA) was used and up to $1 \mu \mathrm{g}$ of total RNA was treated with two units of DNAse I (Invitrogen) in 1X DNase buffer (Invitrogen) in a total volume of $10 \mu \mathrm{l}$ in order to remove genomic DNA. The RT-qPCR was performed in a final volume of $20 \mu \mathrm{l}$, containing $2 \mu \mathrm{l}$ of cDNA, $10 \mu 1$ 2X SYBR Green I reagent, $6.25 \mathrm{U}$ multi-scribe reverse transcriptase, $10 \mathrm{U}$ RNase inhibitor and $0.1 \mathrm{mM}$ primers. The PCR cycling protocol was as follows: $94^{\circ} \mathrm{C}$ for $4 \mathrm{~min} ; 94^{\circ} \mathrm{C}$ for $20 \mathrm{sec}, 55^{\circ} \mathrm{C}$ for $30 \mathrm{sec}$, and $72^{\circ} \mathrm{C}$ for $20 \mathrm{sec} ; 2 \mathrm{sec}$ for plate reading for 35 cycles; and melting curve from $65-95^{\circ} \mathrm{C}$. $\beta$-actin was used as a control for normalizing gene expression. PCR was performed using a Realplex Thermal Cycler (Eppendorf, Hauppage, NY, USA). Experiments were performed independently at least three times and the date analysed using the $\mathrm{R}=2^{-[\Delta \text { Ctsample- }-\Delta \text { Ctcontrol] }}$ formula.

Western blot analysis. Total protein was extracted from ARPE-19 cells using RIPA Cell Lysis Buffer (Bio Rad Laboratories, Inc., Hercules, CA, USA), containing a phosphatase inhibitor and the protease inhibitor cocktail (Sigma-Aldrich), by incubation on ice for $30 \mathrm{~min}$. The cells were then washed with ice-cold phosphate-buffered saline and lysed with RIPA Cell Lysis Buffer (Bio-Rad Laboratories, Inc., Hercules, CA, USA), containing a phosphatase inhibitor and the protease inhibitor cocktail (Sigma-Aldrich), by incubation on ice for $30 \mathrm{~min}$. Lysates were collected by centrifugation at $6,000 \mathrm{xg}$ for $10 \mathrm{~min}$ at $4^{\circ} \mathrm{C}$ and protein concentrations were determined by the BCA protein assay kit (BioTeke, Beijing, China). The samples (30 $\mu \mathrm{g}$ protein/lane) were separated on $10 \%$ SDS-PAGE (Sigma-Aldrich) and transferred onto polyvinylidene fluoride membranes. After blocking in Tris-buffered saline buffer $(50 \mathrm{mmol} / \mathrm{l} \mathrm{NaCl}, 10 \mathrm{mmol} / \mathrm{l}$ Tris, $\mathrm{pH}$ 7.4) containing $5 \%$ nonfat milk, the blots were incubated with primary antibodies against DKK1 (sc-374574; 1:1,500), $\beta$-catenin (sc-53484; 1:3,000), cyclin D1 (sc-20044; 1:3,000) and $\beta$-actin (sc-21733; 1:1,500; Invitrogen; Thermo Fisher Scientific, Inc.) at $4^{\circ} \mathrm{C}$ overnight. Membranes were then washed and incubated with horseradish peroxidase-conjugated secondary antibodies (sc-395760; 1:3,000; Santa Cruz Biotechnology, Inc.). The blots were visualized by super ECL and quantified using Quantity One software (Bio-Rad Laboratories, Inc.).

Cell proliferation. Cell proliferation was analyzed by using cell counting assay kit-8 (CCK-8; Dojindo Molecular Technologies, Inc., Kumamoto, Japan) according to the manufacture's protocol. Cells transfected with Ad-GFP or Ad-DKK1 were cultured in 96-well flat bottomed microplates and were incubated in 10\% CCK- 8 (Dojindo; Kumamoto, Japan) diluted in normal cultured medium for $1 \mathrm{~h}$ at $37^{\circ} \mathrm{C}$. Proliferation rates were determined at 24, 48, 72 and $96 \mathrm{~h}$ after transfection. The absorbance of each well was measured using a microplate reader set at $490 \mathrm{~nm}$. The experiment was repeated three times.

Cell migration assay. RPE cells were grown to confluence in 12-well plastic dishes and were treated with Ad-GFP or Ad-DKK1. RPE cells in $200 \mu$ l serum-free DMEM were added to the upper compartment using an 8- $\mu \mathrm{m}$ microporous filter (EMD Millipore, Boston, MA, USA). Then, $500 \mu 1$ DMEM containing 10\% FBS was added to the bottom chamber. After $24 \mathrm{~h}$ incubation at $37^{\circ} \mathrm{C}$, the cells on the lower surface of the filter were fixed, stained and examined under a light microscope. A total of ten areas were selected randomly from each well, and the cells in three wells from each group were quantified.

Statistical analysis. All experiments were performed independently at least three times. Statistical significance was evaluated using one-way analysis of variance using SPSS software version 10.0 (SPSS, Inc., Chicago, IL, USA). P $<0.05$ was considered to indicate a statistically significant difference.

\section{Results}

DKK1 transfection successfully upregulated the expression of DKK1 in ARPE-19 cells. The effect of the DKK1 transfection on the mRNA and protein expression of DKK1 in ARPE-19 cells was determined using RT-qPCR and western blot analyses. RT-qPCR demonstrated that the overexpression of DKK1 obviously increased DKK1 mRNA levels in ARPE-19 cells compared with control cells (Fig. 1A). Consistent with the results of RT-qPCR, western blot analysis showed that the protein expression levels of DKK1 were significantly increased in DKK1-transfected cells compared with control cells (Fig. 1B). These results indicated that Ad-DKK1 transfection had been successful.

Effect of DKK1 on human RPE cell proliferation. As levels of DKK-1 are lower in PDR patients compared with NPDR patients, we analyzed the effect of DKK1 overexpression on cell proliferation using a CCK-8 assay. As shown in Fig. 2, overexpression of DKK1 significantly suppressed the proliferation of ARPE-19 cells, as compared with the control group. 
A

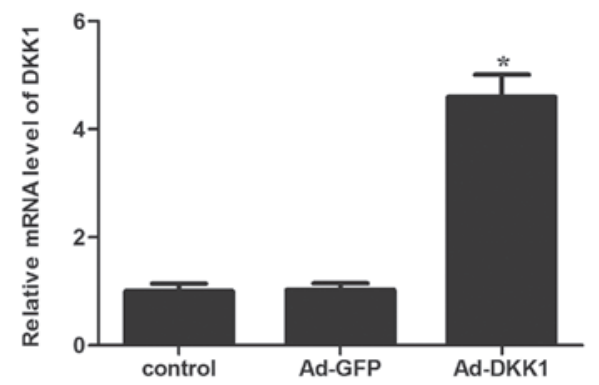

DKK1

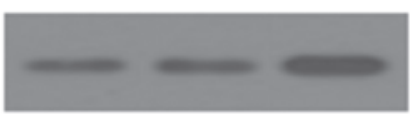

$\beta$-actin
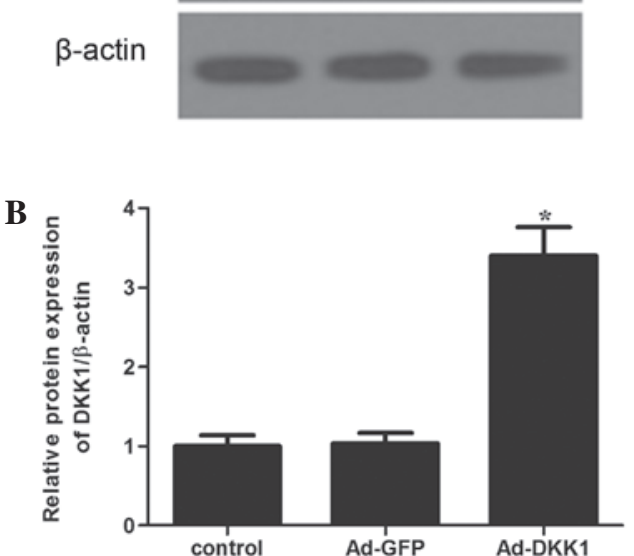

Figure 1. Effect of Ad-DKK1 on DKK1 expression in ARPE-19 cells. (A) Representative images of relative mRNA level of DKK1 transfected with Ad-GFP or Ad-DKK1. (B) Represent western blots of DKK1. Expression levels of proteins were normalized against $\beta$-actin. DKK1 mRNA and protein levels in ARPE-19 cells transduced with Ad-DKK1 were increased when compared with the control group. All experiments were repeated at least three times. " $\mathrm{P}<0.05$ vs. control. DKK1, Dickkopf 1; Ad, adenovirus; GFP, green fluorescent protein.

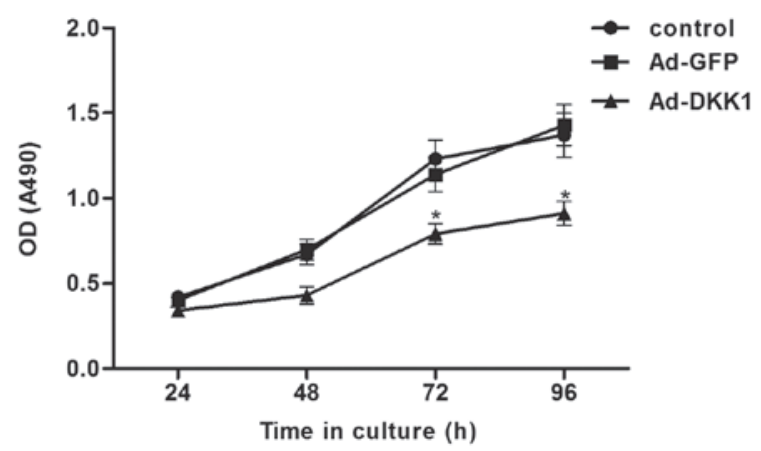

Figure 2. Effect of DKK1 on the proliferation of human ARPE-19 cells. ARPE-19 cells transfected with Ad-GFP or Ad-DKK1 were measured with a CCK-8 assay at 24, 48, 72 and $96 \mathrm{~h}$. DKK1 significantly inhibited the proliferation of ARPE-19 cells when compared with the control groups. All experiments were repeated at least three times. ${ }^{~} \mathrm{P}<0.05$ vs. control. OD, optical density; Ad, adenovirus; GFP, green fluorescent protein; DKK1, Dickkopf 1.

Effect of DKK1 on human RPE cell migration. A Transwell assay was conducted to determine whether DKK1 was involved in the regulation of migration of ARPE-19 cells. As shown in Fig. 3, overexpression of DKK1 significantly reduced the number of migrated cells, as compared with the control group.

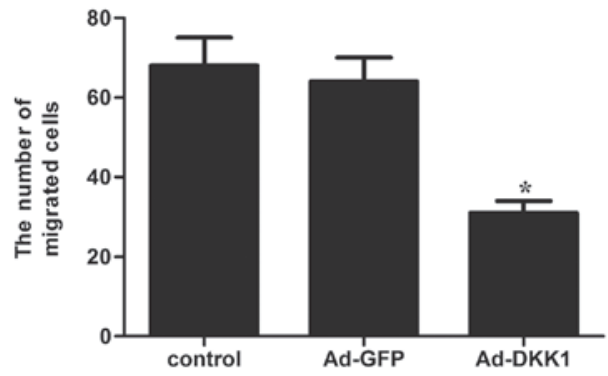

Figure 3. Effect of DKK1 on the migration of human ARPE-19 cells. Transwell assay was performed to evaluate the migration potential of ARPE-19 cells. The number of migrated cells was significantly inhibited in Ad-DKK1-transfeced group when compared with the control groups. All experiments were repeated at least three times. "P<0.05 vs. control. Ad, adenovirus; GFP, green fluorescent protein; DKK1, Dickkopf 1.

A control Ad-GFP Ad-DKK1

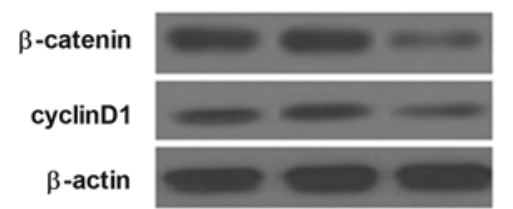

B

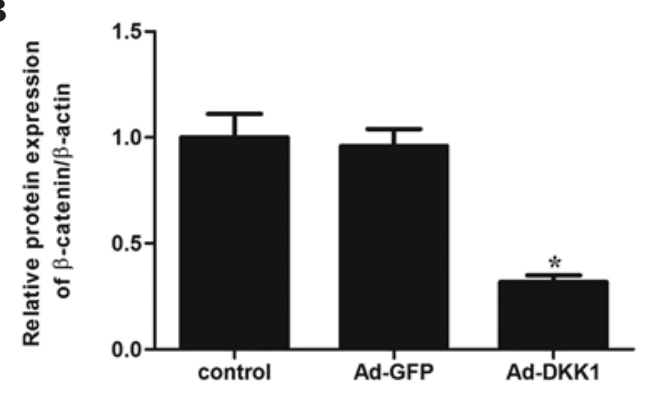

C

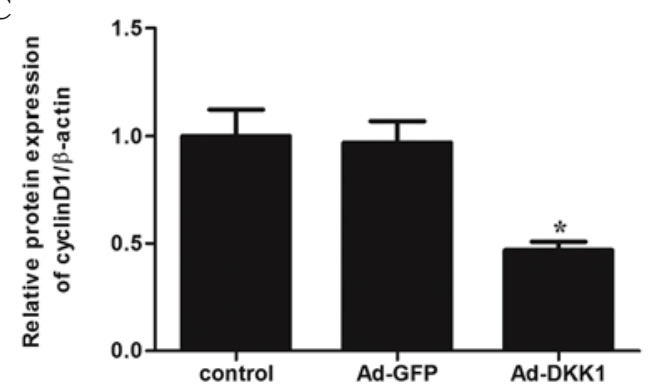

Figure 4. DKK1 inhibits the proliferation and migration of ARPE-19 cells by suppressing the Wnt/ $\beta$-catenin signaling pathway. (A) Levels of $\beta$-catenin and cyclin D1 were detected in control, Ad-GFP and Ad-DKK1-transfected ARPE-19 cells by western blot analysis. Relative protein expression levels of (B) $\beta$-catenin and (C) cyclin D1 were quantified and normalized against $\beta$-actin. All experiments were repeated at least three times. " $\mathrm{P}<0.05$ vs. control. Ad, adenovirus; GFP, green fluorescent protein; DKK1, Dickkopf 1.

Effect of DKK1 on Wnt/ $\beta$-catenin signaling pathway. The Wnt/ $\beta$-catenin signaling pathway was been speculated to be associated with DR. Therefore, we examined the effect of DKK1 on a number of molecules involved in the Wnt $/ \beta$-catenin signaling pathway. Western blot analysis revealed that DKK1 overexpression markedly inhibited the protein expression levels of $\beta$-catenin and cyclin D1 in 
ARPE-19 cells, as compared with the Ad-GFP and control groups (Fig. 4).

\section{Discussion}

The results of the present study suggested that the overexpression of DKK1 significantly inhibited the proliferation and migration of ARPE-19 cells. In addition, the overexpression of DKK1 markedly inhibited the expression of $\beta$-catenin and cyclin D1 in ARPE-19 cells.

RPE cells serve important functions in the healthy eye and under pathological conditions (11). The proliferation of RPE cells plays an important role in the pathogenesis of DR (12). Cell migration is another important process in the development of DR. Without migration, cells are unable to access ectopic sites and form membranes (13). Therefore, the inhibition of RPE cell proliferation and migration may offer a potential route of investigation for developing novel treatments for DR. Previous studies $(14,15)$ found that DKK1 was implicated in the control of cancer cell proliferation and migration. A recent study showed that the overexpression of DKK1 evidently inhibited colon cancer cell proliferation, migration and invasiveness (16). Another study reported that the knockdown of DKK1 using small interfering RNA results in a reduction in intrahepatic cholangiocarcinoma cell migration and invasion (17). In the present study, the overexpression of DKK1 significantly inhibited the proliferation and migration of ARPE-19 cells. These dual roles of DKK1 could attribute to organ-specific actions and different cellular contexts. Collectively, the present results and those of prior studies suggest that DKK1 induces the inhibition of RPE cell proliferation and migration.

The Wnt/ $\beta$-catenin signaling pathway has been widely implicated as the regulator of proliferation and migration in numerous cell types (18-20). $\beta$-catenin is a critical component of the Wnt signaling pathway (21). It has been reported that epidermal growth factor promotes RPE cell proliferation through the $\beta$-catenin signaling pathway (22). DKK1 is a secreted antagonist of the Wnt/ $\beta$-catenin signaling pathway. Koch et al reported that the downregulation of DKK1 increased proliferation of epithelial cells in the large intestine, which was associated with increased transcriptional activity of $\beta$-catenin (23). A prior study showed that FH535, a specific inhibitor of $\beta$-catenin signaling, reduced the outgrowth of cultured RPE sheets and suppressed dedifferentiated RPE cell proliferation and migration (24). Westnskow et al showed that upon $\beta$-catenin deletion, the RPE transforms into a multilayered tissue, and the expression levels of microphthalmia-associated transcription factor and orthodenticle homolog are downregulated, while retina-specific gene expression is increased (25). Consistent with these previous studies, the present results showed that DKK1 overexpression markedly inhibited the expression of $\beta$-catenin and cyclin D1 in ARPE-19 cells, which may lead to reduced RPE cell proliferation and migration.

In conclusion, the present study suggests that overexpression of DKK1 inhibits the proliferation and migration of RPE cells by suppressing the Wnt/ $\beta$-catenin signaling pathway. Therefore, further studies are warranted to investigate the potential for using DKK1 as a therapeutic strategy for DR.

\section{References}

1. Gardner TW, Antonetti DA, Barber AJ, Lanoue KF and Nakamura M: New insights into the pathophysiology of diabetic retinopathy: Potential cell-specific therapeutic targets. Diabetes Technol Ther 2: 601-608, 2000.

2. Pastor JC, de la Rúa ER and Martín F: Proliferative vitreoretinopathy: Risk factors and pathobiology. Prog Retin Eye Res 21: 127-144, 2002.

3. Kawano Y and Kypta R: Secreted antagonists of the Wnt signalling pathway. J Cell Sci 116: 2627-2634, 2003.

4. Krupnik VE, Sharp JD, Jiang C, Robison K, Chickering TW, Amaravadi L, Brown DE, Guyot D, Mays G, Leiby K, et al: Functional and structural diversity of the human Dickkopf gene family. Gene 238: 301-313, 1999.

5. Mikheev AM, Mikheeva SA, Rostomily R and Zarbl H: Dickkopf-1 activates cell death in MDA-MB435 melanoma cells. Biochem Bioph Res Commun 352: 675-680, 2007.

6. Diarra D, Stolina M, Polzer K, Zwerina J, Ominsky MS, Dwyer D, Korb A, Smolen J, Hoffmann M, Scheinecker C, et al: Dickkopf-1 is a master regulator of joint remodeling. Nat Med 13: 156-163, 2007.

7. Wang FS, Ko JY, Yeh DW, Ke HC and Wu HL: Modulation of Dickkopf-1 attenuates glucocorticoid induction of osteoblast apoptosis, adipocytic differentiation and bone mass loss. Endocrinology 149: 1793-1801, 2008.

8. Wang FS, Ko JY, Lin CL, Wu HL, Ke HJ and Tai PJ: Knocking down dickkopf-1 alleviates estrogen deficiency induction of bone loss. A histomorphological study in ovariectomized rats. Bone 40: 485-492, 2007.

9. Qiu F, He J, Zhou Y, Bai X, Wu G, Wang X, Liu Z, Chen Y, Ma JX and Liu Z: Plasma and vitreous fluid levels of Dickkopf-1 in patients with diabetic retinopathy. Eye (Lond) 28: 402-409, 2014.

10. Chen L, Li M, Li Q, Wang CJ and Xie SQ: DKK1 promotes hepatocellular carcinoma cell migration and invasion through $\beta$-catenin/MMP7 signaling pathway. Mol Cancer 12: 157, 2013.

11. Ablonczy Z, Dahrouj M, Tang PH, Liu Y, Sambamurti K, Marmorstein AD and Crosson CE: Human retinal pigment epithelium cells as functional models for the RPE in vivo. Invest Ophthalmol Vis Sci 52: 8614-8620, 2011.

12. Kaczmarek R and Misiuk-Hojło M: Patomechanisms in proliferative vitreoretinopathy. Klin Oczna 113: 64-67, 2011.

13. Kim JH, Park S, Chung $\mathrm{H}$ and Oh S: Wnt5a attenuates the pathogenic effects of the Wnt/ $\beta$-catenin pathway in human retinal pigment epithelial cells via down-regulating $\beta$-catenin and Snail. BMB Rep 48: 525-530, 2015.

14. Koppen A, Ait-Aissa R, Hopman S, Koster J, Haneveld F, Versteeg R and Valentijn LJ: Dickkopf-1 is down-regulated by MYCN and inhibits neuroblastoma cell proliferation. Cancer Lett 256: 218-228, 2007.

15. Chen L, Li M, Li Q, Wang CJ and Xie SQ: DKK1 promotes hepatocellular carcinoma cell migration and invasion through $\beta$-catenin/ MMP7 signaling pathway. Mol Cancer 12: 157-170, 2013.

16. Qi L, Sun B, Liu Z, Li H, Gao J and Leng X: Dickkopf1 inhibits epithelial-mesenchymal transition of colon cancer cells and contributes to colon cancer suppression. Cancer Sci 103: 828-835, 2012.

17. Shi RY, Yang XR, Shen QJ, Yang LX, Xu Y, Qiu SJ, Sun YF, Zhang X, Wang Z, Zhu K, et al: High expression of Dickkopf-related protein 1 is related to lymphatic metastasis and indicates poor prognosis in intrahepatic cholangiocarcinoma patients after surgery. Cancer 119: 993-1003, 2013.

18. Masckauchán TN, Shawber CJ, Funahashi Y, Li CM and Kitajewski J: Wnt/beta-catenin signaling induces proliferation, survival and interleukin-8 in human endothelial cells. Angiogenesis 8: 43-51,2005.

19. Qin X, Zhang H, Zhou X, Wang C, Zhang H, Zhang X and Ye L: Proliferation and migration mediated by Dkk-1/Wnt/beta-catenin cascade in a model of hepatocellular carcinoma cells. Transl Res 150: 281-294, 2007.

20. Yun MS, Kim SE, Jeon SH, Lee JS and Choi KY: Both ERK and $\mathrm{Wnt} /$ beta-catenin pathways are involved in Wnt3a-induced proliferation. J Cell Sci 118: 313-322, 2005.

21. de la Taille A, Rubin MA, Chen MW, Vacherot F, de Medina SG, Burchardt M, Buttyan R and Chopin D: Beta-Catenin-related anomalies in apoptosis-resistant and hormone-refractory prostate cancer cells. Clin Cancer Res 9: 1801-1807, 2003. 
22. Krugluger W, Seidel S, Steindl K and Binder S: Epidermal growth factor inhibits glycogen synthase kinase-3 (GSK-3) and beta-catenin transcription in cultured ARPE-19 cells. Graefes Arch Clin Exp Ophthalmol 245: 1543-1548, 2007.

23. Koch S, Nava P, Addis C, Kim W, Denning TL, Li L, Parkos CA and Nusrat A: The Wnt antagonist Dkk1 regulates intestinal epithelial homeostasis and wound repair. Gastroenterology 141: 259-268, 268.e1-e8, 2011
24. Umazume K, Tsukahara R, Liu L, Fernandez de Castro JP, McDonald K, Kaplan HJ and Tamiya S: Role of retinal pigment epithelial cell $\beta$-Catenin signaling in experimental proliferative vitreoretinopathy. Am J Pathol 184: 1419-1428, 2014.

25. Westenskow P, Piccolo S and Fuhrmann S: Beta-catenin controls differentiation of the retinal pigment epithelium in the mouse optic cup by regulating Mitf and Otx2 expression. Development 136: 2505-2510, 2009. 